beobachtet werden konnte. Die erste dieser Messungen und der kleinere der Werte bei $558{ }^{\circ} \mathrm{C}$, wo die chemische Analyse unzuverlässig war, ist bei den Berechnungen ausgelassen worden.

Abb. 1 zeigt $\ln D^{+}$als Funktion von $T^{-1}$. Trotz der oben erwähnten chemischen Störungen liegt eine lineare Abhängigkeit vor. Das Ergebnis, siehe Tab. 1, wurde in der Gleichung

$$
D^{+}=(0,079 \pm 0,017) \exp \frac{-16060 \pm 330}{R T}
$$

mit den angegebenen wahrscheinlichen Fehlern ${ }^{11} \mathrm{zu}$ sammengefaßt. Hier wird $D^{+}$in $\mathrm{cm}^{2} \mathrm{sec}^{-1}$ und $R$ in cal $\mathrm{Mol}^{-1} \operatorname{grad}^{-1}$ angegeben. Das Meßintervall, 402 bis $636^{\circ} \mathrm{C}$, entspricht fast dem ganzen Gebiet des flüssigen Zustandes (Schmelz- bzw. Siedepunkt des $\mathrm{ZnBr}_{2}$ : 394 bzw. $656{ }^{\circ} \mathrm{C}$ ).
Wie aus dem Verhalten der Leitfähigkeit ${ }^{12}$ zu erwarten ist, ist der Selbstdiffusionskoeffizient von der Temperatur stark abhängig. Eine nähere Diskussion (vgl. Anm. ${ }^{1}$ ) lohnt sich erst, wenn die Leitfähigkeit auch für Temperaturen oberhalb $460{ }^{\circ} \mathrm{C}$ gemessen

Diese Untersuchung ist vom A to m k m mit én finanziell unterstützt worden. A B. A t o m e n e r g i hat kostenlos das radioaktive Zink zu unserer Verfügung gestellt. Wir danken Herrn Professor N. Ryde für sein freundliches Interesse und den Herren Tekn. Lic. E. Berne und A. Lodding, M. A., für wertvolle Diskussionen. Der eine von uns (L.-E. W ALlin) dankt Tek n iska F orskning s råd e t für ein Stipendium.

11 J. Topping, Errors of Observation and their Treatment, Institute of Physics, London 1955, S. 105.

12 Gmelins Handbuch der anorg. Chem. 8. Aufl. 32, 182 [1924].

\title{
Uber die Kantenemission und andere Emissionen des GaN
}

\author{
Von H. G. Grimmeiss und H. Koelmans \\ Aus den Philips-Forschungslaboratorien Aachen und Eindhoven \\ (Z. Naturforschg. 14 a, 264-271 [1959]; eingegangen am 18. Dezember 1958)
}

\begin{abstract}
Es werden die Fluoreszenzeigenschaften des reinen und des mit Lithium, Zink und Magnesium dotierten GaN bei Anregung mit UV-Licht und Kathodenstrahlen in Abhängigkeit der Temperatur untersucht. Mehrere Emissionsbanden wurden beobachtet, von denen die kurzwelligste Bande als Rekombinationsstrahlung freier Elektronen mit freien Löchern gedeutet wird.
\end{abstract}

Unsere Untersuchungen an GaN ergaben, daß diese Verbindung vom Typ $\mathrm{A}^{\mathrm{III}} \mathrm{B}^{\mathrm{V}}$ unter gewissen Bedingungen sowohl mit ultraviolettem Licht, als auch mit Kathodenstrahlen gute Lumineszenzeigenschaften aufweist. Insbesondere konnten in der Nähe der Absorptionskante mehrere Emissionsbanden nachgewiesen werden.

Solche kantennahen Emissionen sind seit längerer Zeit an $\mathrm{ZnO}^{1,2}, \mathrm{ZnS}$ und $\mathrm{CdS}^{3}$ beobachtet worden. Besonders an CdS sind in diesem Zusammenhang ausgedehnte Untersuchungen durchgeführt worden. Während einige Autoren ${ }^{3,4}$ der Ansicht waren, daß die Emission von Exzitonen verursacht würde, konnten andere zeigen, daß diese Lumineszenz durch Störterme hervorgerufen wird ${ }^{5-8}$. Diese Beobachtung wurde von Diemer ${ }^{9}$ bestätigt. Über die Art der Störterme besteht nach wie vor Unklarheit.

1 J. Ewles, Proc. Roy. Soc., Lond. A 167, 34 [1938].

2 J. T. Randall, Trans. Faraday Soc. 35, 1 [1939].

3 F. A. Kröger, Physica 7, 1 [1940].

${ }^{4}$ F. A. Kröger u. H. J. G. Meyer, Physica 20, 1149 [1954].

5 C. C. Klick, Phys. Rev. 89, 274 [1953].

${ }^{6}$ L. R. Furlong, Phys. Rev. 95, 1086 [1954].

7 J. Lambe, Phys. Rev. 98, 985 [1955].

8 J. Lambe u. C. C. Klick, Phys. Rev. 103, 1715 [1956].
Seit kurzem hat man solche kantennahen Emissionen auch an Ge und $\mathrm{Si}^{10}, \mathrm{SiC}^{11}, \mathrm{GaSb}, \mathrm{GaAs}$ und $\mathrm{InP}^{12}$, hervorgerufen durch Trägerinjektion, beobachtet und als direkte Rekombinationsstrahlung von freien Elektronen mit freien Löchern gedeutet.

Die kurzwelligste Emission des GaN zeigt ein anderes Verhalten als die entsprechende an CdS beobachtete Lumineszenz. Ihre Eigenschaften decken sich dagegen weitgehend mit den an Si und Ge beobachteten Emissionen.

\section{Herstellung}

Als Ausgangsmaterial für die Herstellung von $\mathrm{GaN}$ benutzten wir besonders gereinigtes Galliummetall *. Eine spektrochemische Analyse dieses Galliums zeigte folgende atomare Konzentrationen von Fremdelementen: $\mathrm{Hg}=10 \cdot 10^{-6}$ (bedingt durch die Reinigungsmethode),

9 G. Diemer, Physica 23, 987 [1957].

10 J. R. Haynes u. H. B. Briggs, Phys. Rev. 86, 647 [1952].

11 K. Lehovec, C. A. Accardo u. E. Jamgochian, Phys. Rev. 83, 603 [1951].

12 R. Braunstein, Phys. Rev. 99, 1892 [1955].

* Wir danken Herrn Dr. J. J. Engelsmann für die Herstellung des reinen Galliums. 
$\mathrm{Ag}=3 \cdot 10^{-6}, \mathrm{Mn}=2 \cdot 10^{-6}, \mathrm{Al}=3 \cdot 10^{-6}$ und $\mathrm{Si}=$ $2 \cdot 10^{-6}$. Mg, Fe, $\mathrm{Na}$ und $\mathrm{Li}$ konnten nicht nachgewiesen werden. Gute Ergebnisse erhielten wir auch mit dem handelsüblichen reinen Galliummetall. Für unsere Experimente verwendeten wir hauptsächlich ein $\mathrm{GaN}$, das aus $\mathrm{Ga}$ und $\mathrm{NH}_{3}$ hergestellt wurde ${ }^{13-16}$. Das Gallium wurde im $\mathrm{NH}_{3}$-Strom $(0,75 \mathrm{l} / \mathrm{min})$ in einem Quarzrohr erhitzt. Ab etwa $1050{ }^{\circ} \mathrm{C}$ tritt eine heftige Reaktion ein, wobei in wenigen Sekunden das Gallium weitgehend zu $\mathrm{GaN}$ umgesetzt wird. Zur Vervollständigung der Reaktion wurde noch eine Stunde im $\mathrm{NH}_{3}$-Strom auf $1080^{\circ} \mathrm{C}$ erhitzt. Als Reaktionsgefäß wurden Korundschiffchen verwendet. Das $\mathrm{NH}_{3}$ wurde zur Reinigung zuerst aus der Bombe in ein Glasgefäß destilliert und dann in das Reaktionsrohr verflüchtigt. Anfangs trockneten wir das flüssige $\mathrm{NH}_{3}$ mit Li. Es stellte sich jedoch heraus, daß dann bei der Verdampfung des Ammoniaks Li mitgerissen wird, wodurch mit $\mathrm{Li}$ dotierte Präparate entstehen, die eine ziemlich starke gelbe Fluoreszenz zeigen. Deswegen wurde auf eine besondere Trocknung mit Lithium verzichtet. Die aus Gallium und $\mathrm{NH}_{3}$ hergestellten GaNPräparate waren immer hellgrau gefärbt. Es zeigte sich, daß die Reaktionstemperatur nicht viel höher als $1080^{\circ} \mathrm{C}$ sein darf, weil das $\mathrm{GaN}$ sonst wieder allmählich in $\mathrm{Ga}$ und $\mathrm{N}_{2}$ zerfällt.

Nebenbei versuchten wir noch andere Herstellungsmethoden von $\mathrm{GaN}$. Nach $\mathrm{H}_{\mathrm{AHN}}$ und JuzA ${ }^{14}$ wurde $\left(\mathrm{NH}_{4}\right)_{3} \mathrm{GaF}_{6}$ im $\mathrm{NH}_{3}$-Strom auf $900{ }^{\circ} \mathrm{C}$ erhitzt. Das in dieser Weise hergestellte GaN fluoreszierte nicht und war bei den obengenannten Autoren gelb gefärbt. Möglicherweise wird diese gelbe Farbe durch einen geringen Fluoreinbau verursacht.

Weiter wurde $\mathrm{GaN}$ nach JuzA und Hund ${ }^{17}$ durch $\mathrm{Ab}$ bau von $\mathrm{Li}_{3} \mathrm{GaN}_{2}$ im $\mathrm{NH}_{3}$-Strom auf $600{ }^{\circ} \mathrm{C}$ hergestellt. Die in dieser Weise gemachten Präparate waren fast weiß, aber enthielten immer ein wenig Li.

\section{Dotierung}

Fügt man dem fertigen $\mathrm{GaN}$ eine Aktivatorlösung hinzu und erhitzt man nach Trocknung im $\mathrm{NH}_{3}$, dann stellt sich heraus, daß in dieser Weise - auch bei den höchsten Temperaturen, bei denen noch ohne Zersetzung des $\mathrm{GaN}$ gearbeitet werden konnte (ungefähr $1150^{\circ} \mathrm{C}$ ) keine Aktivierung zu spüren war. Eine Aktivierung erreichten wir nur dann, wenn die Aktivatoren als Legierung mit dem $\mathrm{Ga}$ zusammen zur Reaktion gebracht wurden. Dieser Effekt könnte erklärt werden, wenn man annimmt, daß das Gitter des $\mathrm{GaN}$ unterhalb der Zersetzungstemperatur ganz starr ist, und daß daher bei gewöhnlichem $\mathrm{N}_{2}$-Druck die zum Einbau notwendigen Diffusionsgeschwindigkeiten nicht erreicht werden können.

Außer mit $\mathrm{Li}$ wurden auch mit $\mathrm{Mg}$ und $\mathrm{Zn}$ positive Resultate erzielt, die in den folgenden Abschnitten weiter

14 H. Hahn u. R. Juza, Z. anorg. allg. Chem. 244, 111, 133 [1940].

15 J. V. Lirman u. H. S. Zdanov, Acta Phys. Chim. URSS 6, 306 [1937]. diskutiert werden. Wenn wir eine Ga-Zn-Legierung im $\mathrm{NH}_{3}$-Strom erhitzten, wurde beobachtet, daß das $\mathrm{Zn}$ als $\mathrm{Zn}_{3} \mathrm{~N}_{2}$ aus der Legierung verdampfte, ehe das Gallium zu GaN reagiert hatte. Es wurde deshalb das $\mathrm{Zn}$ in ein besonderes Schiffchen außerhalb der Reaktionszone gestellt. In dieser Anordnung war das Gallium während der Reaktion mit einem $\mathrm{Zn}$ beladenen $\mathrm{NH}_{3}$-Strom in Berührung gekommen und das $\mathrm{Zn}$ wurde eingebaut.

\section{Experimentelle Ergebnisse}

\section{Remissionsmessungen}

Remissionsmessungen an mehreren - auch verschieden dotierten - GaN-Proben ergaben, daß der Temperaturverlauf der Absorptionskante zwischen der Temperatur flüssiger Luft und $373^{\circ} \mathrm{K}$ nicht linear zu sein scheint. Bei diesen Untersuchungen wurde der Punkt, bei dem auf der langwelligen Seite der Remissionskurve der lineare Verlauf in einen nicht linearen übergeht, als Remissionskante definiert ${ }^{18}$, wobei wir es offenlassen, inwieweit dadurch die Breite der verbotenen Zone bestimmt ist. Diese wurde bei ca. $90^{\circ} \mathrm{K}$ zu $3,34 \mathrm{eV}$, bei Zimmertemperatur zu $3,26 \mathrm{eV}$ und bei $373^{\circ} \mathrm{K}$ zu $3,22 \mathrm{eV}$ ermittelt.

Alle, auch die dotierten Proben zeigten, abgesehen von einem mit zunehmender Temperatur abnehmenden Pegel der Restabsorption keine weiteren Absorptionsmaxima, obwohl die Meßkurven zunächst mehrere Maxima aufweisen.

Diese Maxima kommen durch Überlagerung der Remission mit der gleichzeitig auftretenden Emission (Lumineszenz) des GaN zustande. Beide Anteile lassen sich dadurch trennen, daß man einmal die Probe mit dem gesamten interessierenden Spektralbereich der Lichtquelle bestrahlt und die Intensität der remittierten Strahlung als Funktion der Wellenlänge bestimmt und ein anderes Mal die Intensität der remittierten Strahlung der mit monochromatischem Licht bestrahlten Probe mißt. Als Vergleichssubstanz benützten wir $\mathrm{MgO}$.

\section{Lumineszenz bei Anregung mit Kathodenstrahlen}

Regt man reines Galliumnitrid mit Kathodenstrahlen $(6 \mathrm{kV})$ an (Abb. 1), dann kann man bei der Temperatur flüssiger Luft eine schmale Emissions-

13 R. Juza u. H. HAHN, Z. anorg. allg. Chem. 239, 282 [1938].

16 E. Kauer u. A. Rabenau, Z. Naturforschg. 12 a, 942 [1957].

17 R. Juza u. F. Hund, Z. anorg. allg. Chem. 257, 13 [1948].

18 E. Kauer u. A. Rabenau, Z. Naturforschg. 13 a, 531 [1958]. 


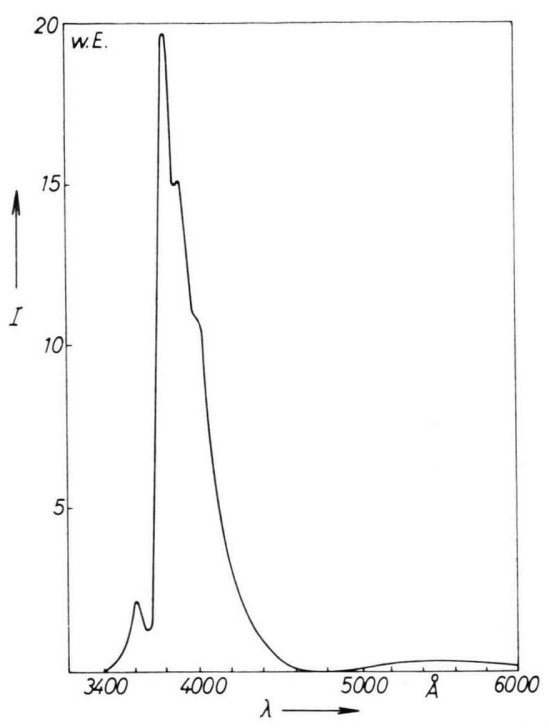

Abb. 1. Emission des reinen $\mathrm{GaN}$ bei ca. $90^{\circ} \mathrm{K}$ mit Kathodenstrahlen.

bande im UV bei $3600 \AA$, drei etwas breitere Emissionsbanden - sogenannte Satelliten - bei $3785 \AA$, $3890 \AA$ und ca. $4050 \AA$ und eine sehr breite Emissionsbande mit einem Maximum bei $5600 \AA$ nachweisen. Während sich bei einer Erhöhung der Temperatur auf ca. $200{ }^{\circ} \mathrm{K}$ (Abb. 2) das Maximum der im UV liegenden Bande nach $3650 \AA$ unter gleichzeitiger starker Intensitätsabnahme verschiebt, bleibt die Lage der Satelliten und die der gelben Emission praktisch unverändert. Eine weitere Temperaturerhöhung auf $293^{\circ} \mathrm{K}$ verlagert das Maximum der kurzwelligsten Emission nach $3710 \AA$, ändert jedoch nichts an der Lage der gelben Emission. Auch bei weiterer Temperaturerhöhung auf $373^{\circ} \mathrm{K}$ und $473^{\circ} \mathrm{K}$ läßt sich keine Verschiebung des Maximums der gelben Emission nachweisen, wohl aber verlagert sich die kurzwelligste Emission zu längeren Wellenlängen hin.

Eine Untersuchung der Intensitäten der einzelnen Emissionsbanden bei verschiedenen Temperaturen (Abb. 3), die aus Gründen der Meßanordnung nur qualitativ durchgeführt wurde, ergab, daß die gelbe Emission mit abnehmender Temperatur zunächst zunimmt, bei ca. $200{ }^{\circ} \mathrm{K}$ ein Maximum hat und dann abnimmt. Die Intensität der kurzwelligsten Emission dagegen nimmt mit abnehmender Temperatur zunächst nur langsam zu, steigt dann aber bei ca. $200^{\circ} \mathrm{K}$ sehr steil an. Dieser rasche Anstieg bei ca. $220^{\circ} \mathrm{K}$ ist bei der $3785 \AA$-Bande noch ausgeprägter. Obwohl sie bei der Temperatur von flüssiger Luft ungefähr zwanzigmal so intensiv wie die gelbe Emission war, konnte sie bei Zimmertemperatur nicht mehr nachgewiesen werden. Ähnliche Verhältnisse gelten für die $3890 \AA$ A-Emission.

\section{Lumineszenz bei Anregung mit UV-Licht}

Die spektrale Energieverteilung unserer Proben zeigte bei Zimmertemperatur und tieferen Tempe. raturen bei Anregung mit UV-Licht gegenüber der Anregung mit Kathodenstrahlen keinen Unterschied. Alle weiteren Untersuchungen wurden deshalb mit UV-Licht durchgeführt. Den Exzitations-Spektren kann man entnehmen, daß sich die maximalen Anregungsenergien nur wenig vom Bandabstand unterscheiden. Um Emissionen nahe der Bandkante noch genau messen zu können, war es notwendig, eine Filterkombination mit möglichst steiler Absorptionskante im kurzwelligen Ausläufer der GaN-Kante zu

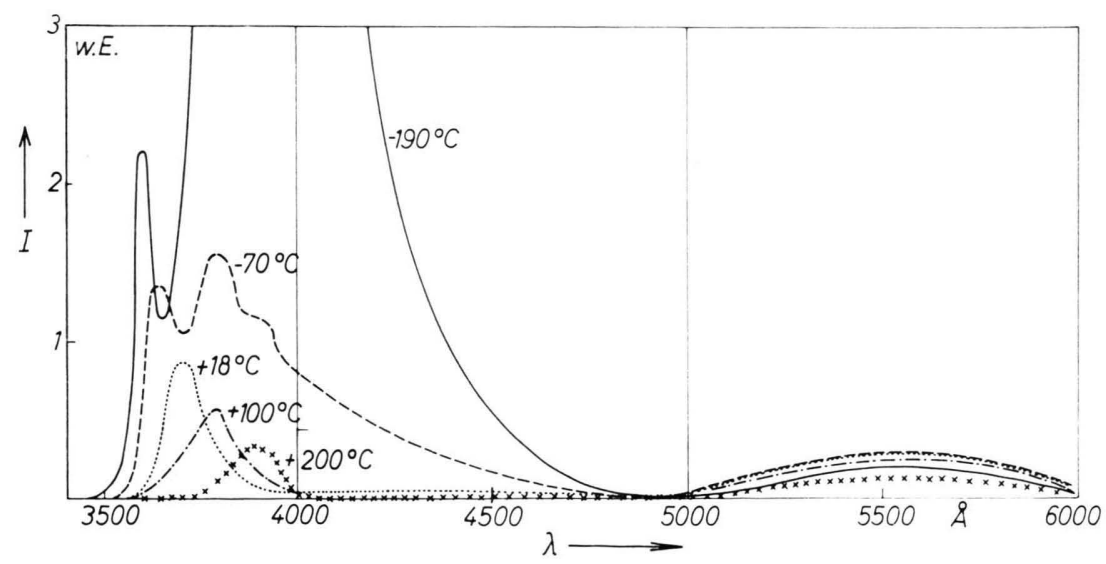

Abb. 2. Die Temperaturabhängigkeit der Emissionen des reinen GaN. 


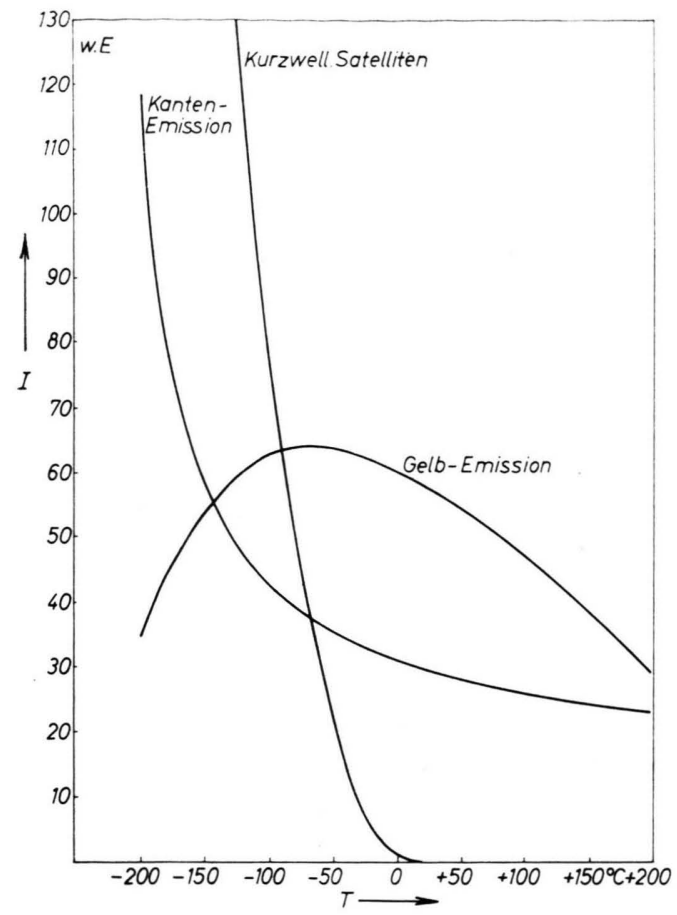

Abb. 3. Die Temperaturabhängigkeit der Intensitäten der am reinen $\mathrm{GaN}$ beobachteten Emissionen.

finden. Bei allen Untersuchungen von Emissionsspektren benutzten wir als Lichtquelle eine HP. Quecksilberlampe $90 \mathrm{~W}$. Als Filter wurde ein $2 \mathrm{~mm}$ Schottfilter UG 11 in Verbindung mit einem KaliumChromat-Flüssigkeitsfilter $\left(150 \mathrm{mg} / \mathrm{l}_{\mathrm{H}_{2} 0}, 10 \mathrm{~mm}\right.$-Küvette) verwendet. Diese Kombination gestattet es, zwischen $3500 \AA$ und $6500 \AA$ selbst unter extremen Bedingungen die Emissionen unserer Proben völlig frei von Strahlungsanteilen der erregenden Lichtquelle zu untersuchen - mit Ausnahme eines kleinen Peaks, hervorgerufen durch die 3650-Linie der Quecksilberlampe, den wir zur Eichung benützten.

\section{Dotierte Proben}

Dotiert man GaN mit Lithium (Abb.4), dann tritt eine wesentliche Verstärkung der gelben Emission ein. - Im Gegensatz zum reinen GaN nimmt jedoch die Intensität dieser gelben Emission bei tieferen Temperaturen nicht ab, sondern je nach Probe bis um den Faktor 5 (bei ca. $90^{\circ} \mathrm{K}$ ) zu. Während die Lage der gelben Emission beim reinen $\mathrm{GaN}$ praktisch unabhängig von der Temperatur ist, verschiebt sich das Maximum beim GaN-Li mit abnehmender Temperatur zu längeren Wellenlängen hin. Diese
Verschiebung kann zwischen Zimmertemperatur und ca. $90^{\circ} \mathrm{K}$ ungefähr $300 \AA$ betragen.

Während man bei der Lithiumdotierung eine Verstärkung der gelben Emission erhält, tritt bei Magnesiumdotierung eine Verstärkung der Satelliten bei

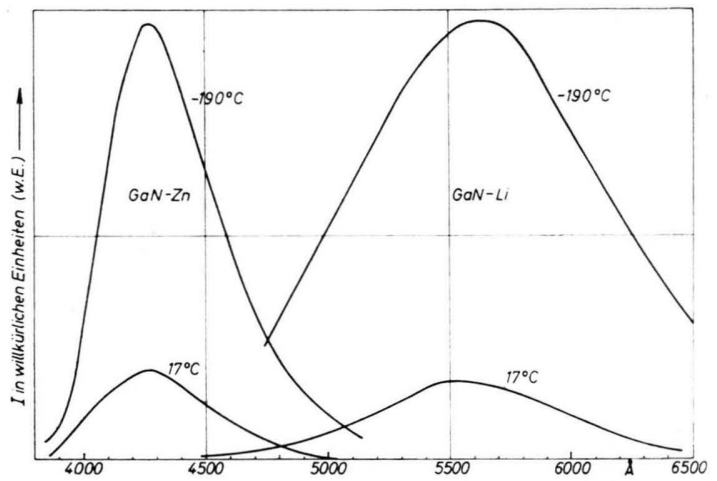

Abb. 4. Die spektrale Verteilung der von Zink und Lithium hervorgerufenen Emissionen bei ca. $90{ }^{\circ} \mathrm{K}$ und $300{ }^{\circ} \mathrm{K}$.

sonst gleicher spektraler Energieverteilung ein. Die Intensitätszunahme kann das zehnfache der Intensität betragen, die man ohne Magnesiumdotierung erhält. Die Lage und Form der Satelliten wird durch die Dotierung nicht beeinflußt. Die gleichen Ergebnisse erhält man, wenn man an Stelle von $\mathrm{Mg}$ mit Indium dotiert.

Dotiert man GaN mit Zink, dann tritt eine neue, breite Emissionsbande mit einem Maximum bei $4300 \AA$ auf (Abb. 4). Die Lage des Maximums ist weitgehend temperaturunabhängig. Die Intensität nimmt mit abnehmender Temperatur zu. Bemerkenswert ist noch, daß bei allen Zn-dotierten Präparaten keine Satellitenemissionen auftreten.

In Abb. 5 geben wir die Ergebnisse wieder, die man erhält, wenn die Intensität der anregenden Strahlung variiert wird. Untersucht wurde die kurzwelligste Emission bei der Temperatur flüssiger Luft und $373{ }^{\circ} \mathrm{K}$, die Satellitenemission bei der Temperatur flüssiger Luft und die gelbe Li-Emission $\left(5600 \AA\right.$ ) bei $373{ }^{\circ} \mathrm{K}$. Die gelbe Emission und die Kantenemission bei beiden Temperaturen zeigen innerhalb unseres Meßbereiches eine lineare Abhängigkeit in der log-log-Darstellung. Die Satellitenemission dagegen ist nur bei kleineren Anregungsdichten linear und geht bei starker Anregung in einen sublinearen Bereich über. Die maximale Anregungsdichte ist bei unserer Meßanordnung durch eine $90 \mathrm{~W}$ HP-Quecksilberlampe gegeben, die mit Hilfe eines Spiegels auf die Probe abgebildet wird. 


\section{Anregungsspektren}

Die Energie lokalisierter Terme innerhalb der verbotenen Zone zwischen dem Valenz- und Leitfähigkeitsband und die des Bandabstandes lassen sich manchmal dadurch bestimmen, daß man die Intensität einer bestimmten Emissionsbande als Funktion

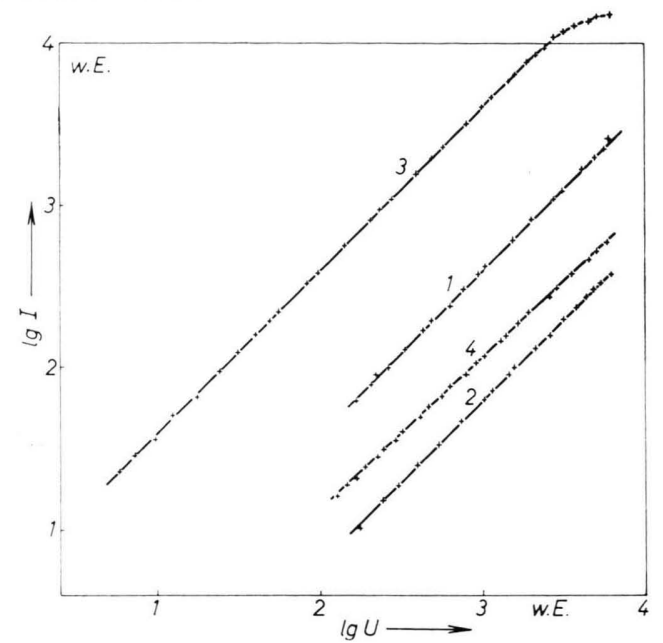

Abb. 5. Die Intensitätsabhängigkeit des $\mathrm{GaN}$ von der Anregungsdichte bei verschiedenen Temperaturen (1 Kantenemission bei ca. $90^{\circ} \mathrm{K}, 2$ Kantenemission bei $373^{\circ} \mathrm{K}, 3$ Satellitenemission bei ca. $90^{\circ} \mathrm{K}, 4$ gelbe $\mathrm{Li}$-Emission bei $373^{\circ} \mathrm{K}$ ).

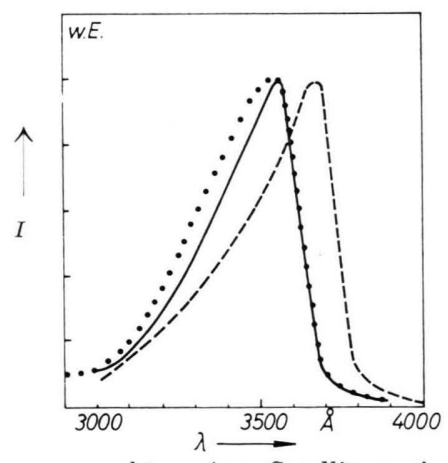

Abb. 6. Anregungsspektren ( $\cdots$ Satellitenemission (3900 Å) des $\mathrm{GaN}-\mathrm{Mg}$ bei ca. $90^{\circ} \mathrm{K}$; — Blauemission (4300 Å) des GaN-Zn bei ca. $90^{\circ} \mathrm{K}$; - - - Gelbemission (5600 Å) des GaN-Li bei Zimmertemperatur).

der Wellenlänge des die Probe anregenden Lichtes mißt. Wir haben die Messungen an Mg-, Zn- und Li-dotierten Proben bei ca. $90^{\circ} \mathrm{K}$ und Zimmertemperatur mit einer Wasserstofflampe als Lichtquelle durchgeführt (Abb. 6). Sämtliche Emissionen setzen bei gleichen Temperaturen bei derselben Energie der erregenden Strahlung ein. Weitere Anregungsmaxima konnten nicht nachgewiesen werden.

Als Einsatzpunkt der Emissionen definierten wir den Schnittpunkt der geradlinigen Verlängerung der langwelligen Anregungskante mit der Abszisse. Für die Satelliten-Emission von $\mathrm{GaN}-\mathrm{Mg}$ und die blaue Emission (4300 $\AA$ ) von $\mathrm{GaN}-\mathrm{Zn}$ ergab sich bei der Temperatur flüssiger Luft jeweils ein Wert von $3,36 \mathrm{eV}$, während sich bei Zimmertemperatur für die gelbe Emission (5600 $\AA$ ) von GaN-Li ein Wert von $3,26 \mathrm{eV}$ ergab.

\section{Abklingzeiten}

Die Abklingzeiten von vier verschiedenen Emissionen wurden bei der Temperatur der flüssigen Luft bestimmt. Die Ergebnisse werden in Tab. 1 wiedergegeben.

\begin{tabular}{|l|c|c|}
\hline Probe & Emission $\AA$ & Abklingzeit $\mu$ sec \\
\hline GaN & 3600 & $\leqq 0,5$ \\
GaN-Li-F & 3800 & $<1$ \\
GaN-Zn & 4300 & 4 \\
GaN-Li & 5600 & 10 \\
\hline
\end{tabular}

Tab. 1.

\section{Kantenemission}

Bei allen untersuchten Proben fanden wir bei ca. $90{ }^{\circ} \mathrm{K}$ im UV sowohl bei Anregung mit Kathodenstrahlen als auch mit UV-Licht (meistens $3131 \AA$ ) eine schmale Emissionsbande, deren Intensität von Probe zu Probe sehr unterschiedlich war. Die größte Intensität bekamen wir jedoch an einer Probe, die die größte Reinheit hatte. Diese bei tiefen Temperaturen ziemlich schmale Bande verbreiterte sich mit steigender Temperatur und verlagerte sich gleichzeitig nach längeren Wellenlängen. In Abb. 7 geben wir die Lage dieser Emission bezüglich der Remissionskante bei verschiedenen Temperaturen wieder.

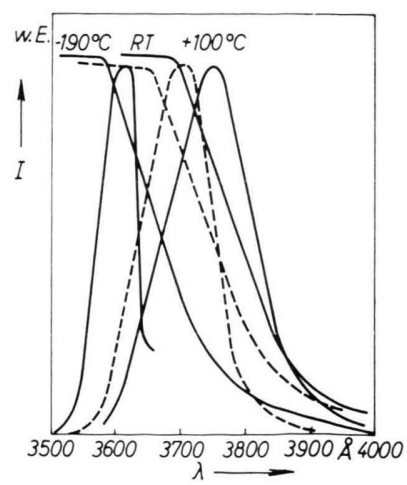

Abb. 7. Synoptische Darstellung der Remissionskante und Kantenemission bei verschiedenen Temperaturen. 
Wie man sieht, liegt das Maximum der Emission jeweils am kurzwelligen Ende der Remissionskante. Dieses Maximum liefert wegen der Selbstabsorption über die wirkliche Lage des Emissionsmaximums keine Aussage. In den meisten Fällen, besonders an

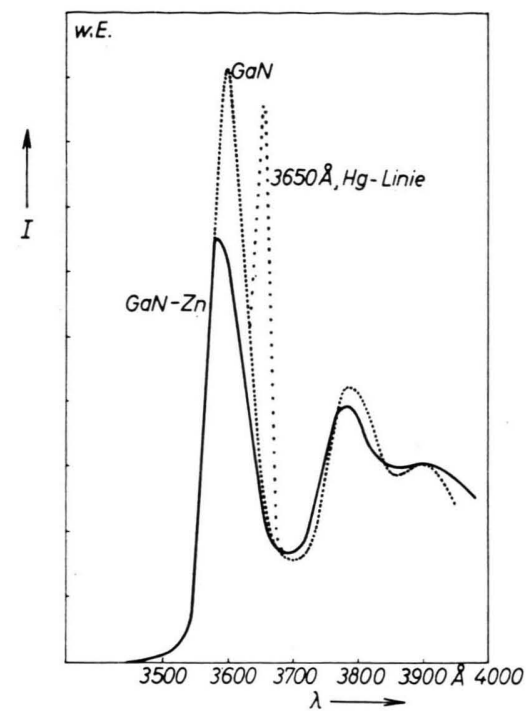

Abb. 8. Kantenemission des reinen $\mathrm{GaN}$ und des $\mathrm{GaN}-\mathrm{Zn}$ bei ca. $90^{\circ} \mathrm{K}$.

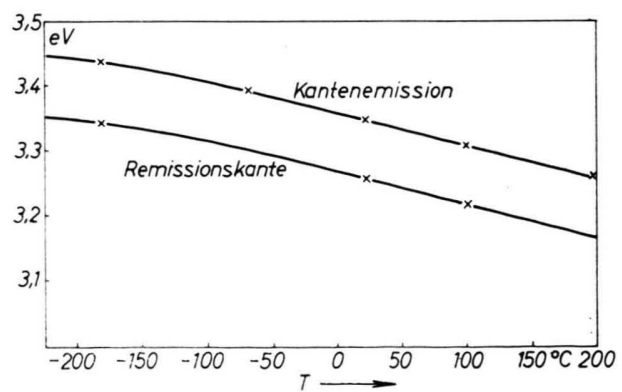

Abb. 9. Temperaturabhängigkeit der Kantenemission und der Remissionskante.

sehr reinen Proben, liefern die Meßergebnisse eine symmetrische Bande. Bei Zn-dotierten Proben konnten wir jedoch eine ausgesprochene Unsymmetrie feststellen (Abb. 8).

In Abb. 9 haben wir die Lage dieses relativen Emissionsmaximums und die Lage der oben definierten Remissionskante als Funktion der Temperatur aufgetragen. Wie man theoretisch wegen der $\mathrm{Ab}$ nahme der Störung der Bänder durch die Gitterschwingungen zu erwarten hat, ist der Verlauf beider Kurven bei tiefen Temperaturen nicht linear. Bemerkenswert ist, daß die Kurven parallel zueinander liegen. Der Temperaturkoeffizient für beide Kurven ändert sich zwischen $2,6 \cdot 10^{-4} \mathrm{eV} / \mathrm{Grad}$ bei $90{ }^{\circ} \mathrm{K}$ und $5,0 \cdot 10^{-4} \mathrm{eV} / \mathrm{Grad}$ bei $473{ }^{\circ} \mathrm{K}$.

\section{Diskussion}

Im folgenden versuchen wir, eine Erklärung für die einzelnen Emissionsbanden zu geben. Bis jetzt sind wir, wie wir bereits oben erwähnten, noch nicht in der Lage, über die Höhe der Dotierung und Verunreinigung quantitative Angaben zu machen.

Die Lage der kurzwelligsten Emission deutet offenbar darauf hin, daß es sich um eine direkte Rekombination freier Elektronen mit freien Löchern handeln kann, wie man sie auch durch Trägerinjektion ${ }^{10-12}$ an anderen Stoffen gefunden zu haben glaubt. Bei einer solchen Kantenemission sollte man erwarten, daß - wenn man von zusätzlichen Phononen-Wechselwirkungen absieht, weil diese nur einige Hundertstel Elektronenvolt betragen können - sie niemals energetisch kleiner als der Bandabstand ist, d. h. sie sollte frühestens bei der Absorptionskante auftreten und sich von dort nach kürzeren Wellenlängen hin erstrecken. Mit zunehmender Energie der Strahlung vergrößert sich aber auch der Absorptionskoeffizient, der so groß werden kann, daß jede entstehende Strahlung wieder absorbiert wird. Eine Kantenemission sollte also an der Absorptionskante einsetzen, wobei ihre Intensität nach kürzeren Wellenlängen hin zunächst zu- und dann wieder abnimmt.

Abb. 7 zeigt, daß unsere kurzwelligste Emission diesen Erwartungen entspricht.

Braunstein ${ }^{12}$ hat an $\mathrm{GaSb}$ und GaAs durch Trägerinjektion ebenfalls eine Emission gefunden, die er als direkte Rekombination von Elektron - LochPaaren deutet. Diese Emissionen sind jedoch im Falle des GaSb um 0,05 eV und im Falle des GaAs um $0,25 \mathrm{eV}$ gegenüber der Absorptionskante nach längeren Wellenlängen hin verschoben. Diese Diskrepanz kann von BRAUnstein nicht erklärt werden. Wir glauben nicht, daß es sich in diesem Fall tatsächlich um eine Kantenemission handelt. Ähnliche Betrachtungen gelten für die an $\mathrm{SiC}$ gefundenen Emissionen.

Haynes und Westrhal ${ }^{19}$ haben ähnliche Untersuchungen wie Braunstein an Silicium durchgeführt. Sie finden ebenfalls in Kantennähe eine Emission, die aber im Gegensatz zum GaAs und $\mathrm{GaSb}$ um 0,02

19 J. R. Haynes u. W. C. Westphal, Phys. Rev. 101, 1676 [1956]. 
(eV) gegenüber der Absorptionskante nach kürzeren Wellenlängen verschoben ist, wie man es für eine Kantenemission erwarten sollte. Auch die anderen Eigenschaften dieser Emission decken sich mit unseren Beobachtungen an GaN.

Würde man annehmen, daß die kurzwelligste Emission des $\mathrm{GaN}$ eine Rekombinationsstrahlung über einen Störterm ist, dann müßte dieser Term wegen des kurzwelligen Charakters der Emission sehr nahe am Valenz- bzw. Leitfähigkeitsband liegen. Die KLasens-Schönsche Modellvorstellung ${ }^{20,21}$ fordert in diesem Fall eine Temperaturlöschung (quenching) schon bei sehr tiefen Temperaturen. Wie man Abb. 3 entnehmen kann, findet jedoch eine solche Temperaturlöschung bis $473^{\circ} \mathrm{K}$ nicht statt.

Weiterhin sollte eine Kantenemission bei Veränderung der Temperatur mit der Bandkante wandern. In Abb. 9 haben wir gezeigt, daß diese Forderung voll erfüllt wird. Die Lage der anderen Emissionen des GaN zeigt demgegenüber entweder keine Temperaturabhängigkeit oder eine solche mit umgekehrten Vorzeichen, wie z. B. GaN-Li.

Ein weiteres Argument für eine Kantenemission sehen wir in der linearen Abhängigkeit der Emission mit der Anregungsdichte (Abb. 5). Diemer ${ }^{9}$ konnte im Falle des CdS zeigen, daß es sich bei der grünen Emission des CdS wegen der gefundenen Sublinearität nicht um eine Kantenemission handeln kann.

In diesem Sinne ist auch die Tatsache zu werten, daß die Kantenemission eine so kleine Abklingzeit hat, daß sie von uns nicht mehr bestimmt werden konnte und daß die Emission besonders stark ist, wenn das $\mathrm{GaN}$ sehr rein ist.

Eine weitere Stütze für unsere Vermutung sehen wir darin, daß die im reinen $\mathrm{GaN}$ symmetrische Bande der Kantenemission eine unsymmetrische Form bekommt, wenn die Probe mit Zink dotiert ist. Der Grund für dieses Verhalten ist unseres Erachtens darin zu sehen, daß die für die blaue Emission verantwortlichen Terme nur wenig über dem Valenzband liegen und demzufolge bevorzugt an der Auffüllung der Löcher im Valenzband beteiligt sind ${ }^{21}$. Bei intrinsinischer Anregung entsteht dadurch im Valenzband eine Verarmung an Löchern. Das bedeutet aber für die Kantenemission eine Intensitätsabnahme, die sich wegen der Selbstabsorption jedoch nur in einer Unsymmetrie der Bande äußern kann.

20 H. A. KLASENS, Nature, Lond. 158, 306 [1946].

21 M. Sснӧм, Z. Phys. 119, 463 [1942] ; Ann. Phys., Lpz. (6) 3, 333 [1948].
Es wäre noch die Frage zu klären, ob es sich bei der Remissionskante tatsächlich um eine BandBand-Anregung handelt. Darüber dürfte jedoch kein Zweifel bestehen, wenn man u. a. bedenkt, daß die verschiedenen Emissionen stets bei der gleichen Energie der erregenden Strahlung einsetzen (Abb. 6) und dieser Einsatzpunkt nicht nur mit der Remissionskante zusammenfällt, sondern auch den gleichen Temperaturkoeffizienten aufweist wie diese.

Wir sind also der Ansicht, daß sich die kurzwelligste Emission des $\mathrm{GaN}$ nicht mit den kantennahen Emissionen des $\mathrm{CdS}, \mathrm{ZnS}$ oder $\mathrm{ZnO}$ vergleichen läßt. Außer den bereits angeführten Argumenten wäre hierzu noch zu erwähnen, daß z. B. beim CdS die grüne Emission eine ausgesprochene Struktur zeigt, während wir eine solche niemals bei der Kantenemission des $\mathrm{GaN}$ feststellen konnten. Außerdem verschwinden die kantennahen Emissionen beim CdS mit steigender Temperatur sehr rasch, während beim $\mathrm{GaN}$ die Kantenemission noch bei $473^{\circ} \mathrm{K}$ gut zu besbachten ist. Im Gegensatz zum CdS, wo die grüne Emission eine entgegengesetzte Verschiebung bei Änderung der Temperatur zeigt wie die Absorptionskante $^{5}$, beobachten wir beim $\mathrm{GaN}$ eine Temperaturabhängigkeit der Kantenemission, die parallel mit der der Absorptionskante verläuft.

Eine Ähnlichkeit der grünen Emission beim CdS scheint nach unserer Auffassung dagegen mit den von uns beobachteten Satelliten zu bestehen. Trotz eingehender Versuche sind auch wir nicht in der Lage, eine Erklärung für die Satelliten zu geben, die zunächst so lange auf Schwierigkeiten stoßen wird, so lange es nicht möglich ist, die Höhe der Dotierungen anzugeben.

Die Satelliten traten besonders stark bei zementgrauen Pulvern auf, die ihrerseits wieder eine um so bessere elektrische Leitfähigkeit zeigten, je dunkler sie waren. Durch vergleichende Messungen an $\mathrm{GaN}$ Einkristallen glauben wir annehmen zu können, daß die dunkle Farbe durch einen Überschuß an eingebautem Gallium verursacht wird. Für diese Annahme würde sprechen, daß die Satellitenemissionen durch Magnesium begünstigt werden. Nicht einzusehen dagegen ist, warum bei zinkdotierten Proben die Satelliten nicht beobachtet wurden, während eine $\mathrm{Li}$ thium-Dotierung keinen Einfluß zu haben scheint. An der Klärung dieser Frage wird z. Zt. noch gearbeitet.

Als Ursache der blauen Emission mit einem Maximum bei $4300 \AA$ ist eindeutig die Zinkdotierung an- 
zusehen. Ohne bewußte Zinkdotierung haben wir bei keiner GaN-Probe eine Emission bei $4300 \AA$ feststellen können.

Dasselbe gilt für die Lithiumdotierung, die sich gegenüber der gelben Emission des reinen $\mathrm{GaN}$, über deren Ursache wir noch nichts sagen können, in völlig anderer Weise verhält. Da wir weder durch Anregungsspektren noch durch Remissionsmessungen die für die verschiedenen Emissionen verantwort- lichen Terme bestimmen konnten, nehmen wir an, $\mathrm{da}$ es sich jeweils nur um sehr geringe Dotierungen handelt.

Für wertvolle und anregende Diskussionen sind wir den Herren Dr. H. A. Klasens, Dr. G. Diemer, Dr. W. Hoogenstraaten, Dr. E. Kauer und Dr. A. Rabenau zu besonderem Dank verpflichtet. Herrn Dr. A. BriL danken wir vielmals für die Messung der Abklingzeiten.

\title{
Elektronenmikroskopische Untersuchung elektrolytisch erzeugter Siliciumoxydschidhten
}

\author{
Von A. Politycki und E. Fuchs \\ Mitteilung aus dem Werkstoff-Hauptlaboratorium der Siemens \& Halske AG \\ (Z. Naturforschg. 14 a, 271-275 [1959]; eingegangen am 6. November 1958)
}

\begin{abstract}
Durch anodische Oxydation auf Silicium erzeugte Deckschichten lassen sich im trockenen Chlor. wasserstoffstrom bei höherer Temperatur von der Unterlage abtrennen und dadurch der elektronenmikroskopischen Untersuchung zugänglich machen. Die in Borsäure hergestellten Filme bestehen aus zwei Schichten, von denen eine stark porös ist. Sie sind den beim Aluminium bekannten Eloxalfilmen ähnlich. Der Porendurchmesser beträgt etwa 100-200 Å.

Im Parallelversuch gelang es, die nach dem chemischen Polieren des Halbleiters an der Luft entstehende Oxydbelegung zu isolieren. Sie ist mit Sicherheit dünner als $100 \AA$ und weist keinerlei charakteristische Risse oder Poren auf.
\end{abstract}

\section{Zur anodischen Oxydation von Silicium}

Die anodische Oxydation des Siliciums wurde offenbar zum ersten Mal von GüNtherschulze und $\mathrm{BETZ}^{1}$ versucht. Die Autoren geben an, daß in Schwefelsäure nur eine unvollständige Formierung möglich war. Neuere Untersuchungen mit definiertem Silicium (einkristallines p-Silicium und n-Silicium von $2-5 \Omega \mathrm{cm}$ ) haben Schmid und Michel ${ }^{2}$ beschrieben. In Salpetersäure und Phosphorsäure ließ sich der Halbleiter bei konstanter Stromdichte auf 80 bzw. 180 Volt formieren, während in einer Lösung von $0,4 \mathrm{~g} / l$ Kaliumnitrat in Methylacetamid sogar eine Spannung von 560 Volt erreicht wurde. Sie fanden, daß sich das oxydbedeckte Silicium ähnlich Aluminium oder Tantal wie ein elektrolytischer Gleichrichter verhält. Bei überschußleitendem Material (n-Silicium) war der Sperreffekt größer als bei defektleitendem Material. Dieser Unterschied läßt sich dadurch erklären, daß die Elektronen beim Anlegen einer positiven Spannung an den Halbleiter abgesaugt werden. Für den Stromtransport kommen

\footnotetext{
1 A. Güntherschulze u. H. Betz, Elektrolytkondensatoren, Techn. Verl. H. Cram, Berlin 1952.
}

in diesem Falle nur Defektelektronen in Betracht, die aber beim n-Silicium praktisch kaum vorhanden sind. Der Verarmungseffekt läßt sich wesentlich verringern, wenn man die Probe stark mit weißem Licht bestrahlt und dadurch ständig zusätzliche ElektronenLochpaare erzeugt. Dann kann der resultierende Stromfluß den Dunkelstrom um Größenordnungen überschreiten, so daß sich der elektrolytische Vorgang durch die Lichteinstrahlung steuern läßt. UHLIR ${ }^{3}$ hat gezeigt, wie man auf elektrolytischem Wege mit Hilfe eines Lichtstrahles tiefe Gruben in Silicium bohren kann.

Ganz ähnlich bildet sich auf n-Silicium bei Verwendung eines passenden Elektrolyten an belichteten Stellen bevorzugt Oxyd. Soll eine gleichmäßige Bedekkung der Oberfläche erzielt werden, ist es daher wichtig, die Probe gut auszuleuchten. Man kann sie z. B. rotieren lassen.

Eine zweckmäßige Anordnung, wie sie bei den im folgenden beschriebenen Versuchen benutzt wurde, zeigt Abb. 1. Die elektrolytische Zelle besteht aus einem offe-

\footnotetext{
2 P. F. Schmidt u. W. Michel, J. Electrochem. Soc. 104, 230 [1957].

3 J. UhliR, Bell Syst. Techn. J. 35, 333 [1956].
} 